\title{
Foreword
}

\section{The Sodium Channel: One Gene, Many Diseases!}

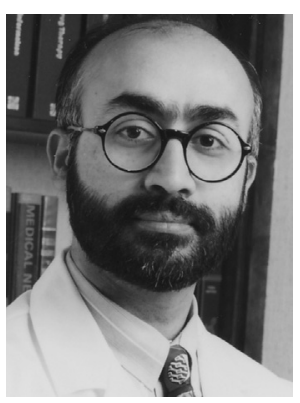

Ranjan K. Thakur, MD, MPH, MBA, FHRS

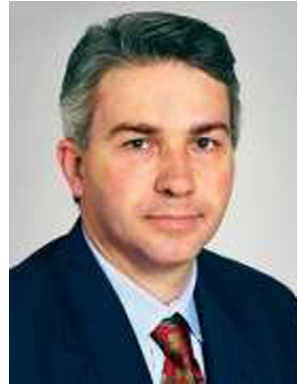

Andrea Natale, MD, FACC, FHRS

Consulting Editors

The cardiac $\mathrm{Na}^{+}$channel is a voltage-gated channel that consists of assemblies of proteins: $\alpha$-subunit, the pore-forming component, and one or two $\beta$-subunits, which modulate the function of the pore or channel. The structure of these proteins is encoded by genes.

A mutation is defined as any change in a DNA sequence away from normal. This implies that there is a normal allele that is prevalent in the population and the mutation changes it to a rare and abnormal variant. In contrast, genetic polymorphism is a DNA variation that is "common" in the population. The arbitrary cutoff point between a mutation and a polymorphism is $1 \%$. Mutations occur in less than $1 \%$ of the population, whereas genetic polymorphisms are defined as variants that occur at a frequency greater than $1 \%$.

Polymorphisms may be caused by insertion/ deletion, single-nucleotide polymorphisms, or short-simple-sequence repeats. Polymorphisms located within the coding region of a gene can directly influence the structure and function of its protein, whereas changes within the regulatory sequences of a gene can influence the level of expression of the protein. Genetic variations may also alter phenotypic expression under certain conditions, such as electrolyte disturbance, ischemia, and so on. However, DNA variation may also be inconsequential and may not have a clinical manifestation.

The $\mathrm{Na}^{+}$channel plays a critical role in cardiac conduction because it initiates the action potential $\left(I_{\mathrm{Na}}\right)$ and, via gap junctions, determines the velocity of conduction and impulse propagation. Mutations/polymorphisms in these genes underlie many clinical arrhythmias and conditions, such as the Brugada syndrome, long QT syndrome, sudden infant death syndrome, conduction abnormalities, sick sinus syndrome, dilated cardiomyopathy, and atrial fibrillation.

Molecular understanding of arrhythmias is proceeding at a breakneck pace, and most clinical electrophysiologists don't have a comprehensive understanding of this field. This is due in large part to a lack of direct translation of molecular understanding into daily practice of clinical medicine. But this is the next frontier.

We want to congratulate Dr Abriel for editing this issue of the Cardiac Electrophysiology Clinics and assembling contributors leading this field to give us expert summaries of the important advances to date. This issue of the Cardiac Electrophysiology Clinics will be of interest to clinical electrophysiologists as well as experts 
in the field who may need to catch up with advances outside of their own focused research interest.

Ranjan K. Thakur, MD, MPH, MBA, FHRS Sparrow Thoracic and Cardiovascular Institute Michigan State University 1200 East Michigan Avenue; Suite 580 Lansing, MI 48912, USA
Andrea Natale, MD, FACC, FHRS Texas Cardiac Arrhythmia Institute Center for Atrial Fibrillation at St. David's Medical Center 1015 East 32nd Street, Suite 516 Austin, TX 78705, USA

E-mail addresses: thakur@msu.edu (R.K. Thakur) andrea.natale@stdavids.com (A. Natale) 